\title{
Shock analysis method for systematic performance evaluation of component embedded in handheld electronic devices
}

\author{
C.S. Chin \\ Research Robotic Centre, Department of Mechanical and Aerospace Engineering, Nanyang Technological \\ University, Singapore 639798, Singapore \\ E-mail: mcschin1@yahoo.com
}

Received 21 February 2005

Revised 20 June 2005

\begin{abstract}
It is important to identify the robustness of product (or embedded component inside the product) against shock due to free drop. With the increasing mobile and fast-paced lifestyle of the average consumer, much is required of the products; such as consumers expect mobile products to continue to operate after drop impact. Since free drop test is commonly used to evaluate the robustness of small component embedded in MP3 player, it is difficult to produce a repeatable shock reading due to highly uncontrolled orientation during the impact on ground. Hence attention has been focus on shock table testing, which produces a higher repeatable result. But it failed to demonstrate the actual shock with the presence of rotational movement due to free drop and also it suffers from a similar limitation of repeatability. From drop to drop, shock tables can vary about $\pm 5 \%$ in velocity change but suitable for making a consistent tracking the product improvement.

Although many test procedure (such as ASTM D3332 [5]) have been written around shock test machines, as a whole, the test perform on the shock tables have no direct correlation over the free-drop testing. Since both will have some velocity change variability. Some products react more to torsion forces than they do to orthogonal forces, fortunately, due to the size of the product, the exposure to this torsion force is less prominent. Since the intent of lab testing is to re-create real world events, a unique ways to establish a shock test that encompasses the features of the two tests are needed. Thus, establishing the robustness of the small component against shock due to topple drop at various tilt angle under different drop orientations and platform (such as on the office table), on a MP3 player is needed. This eventually enables a high repeatable and realistic shock testing for product performance evaluation in MP3 player.

There are few advantages of using tilt tester for shock testing. Firstly, close approximations to the actual free drops test with better repeatability. Secondly, it ensures that the shock level is reduced prior to performing a final free drop test (using actual product) and hence reduces the cost. Thirdly, it reduces the number of trials and cycle time needed for shock testing. Lastly, it provides a platform for shock absorber design for component (embedded in the MP3 player) as shown in the Section 3.
\end{abstract}

Keywords: Shock table, tilt tester, free drop, modeling, performance evaluation

\section{Introduction}

Portable electronics continue to evolve with technology diminishing their size and most notable are MP3 players or even PDAs and cell phones. A fundamental problem still remains for all such product is unable to withstand shock due to free drop on any platform such as plywood, vinyl tile and concrete. Shock is a major cause of damage and failure of portable or handheld electronic devices that incur high cost due to the product failure. In this study, the tilt tester is used to evaluate the performance of the component with shock absorber to ensure improvement (with the shock absorber) is achieved before conducting a final confirmation free drop test. 
In Section 2, a dynamic model of the free drop and tilt tester using energy conservation equation and Newton's second law is derived. To validate these models, test is performed on the component (free drop on a plywood platform). The free drop results are correlated with the tilt test results by adjusting parameters such as spring stiffness and tilt angle of the tilt tester.

\section{Definitions and derivation of shock}

\subsection{Definition of shock}

Shock is typically considered a short duration event with large-magnitude acceleration often expressed as G level where $1 \mathrm{G}=9.81 \mathrm{~m} / \mathrm{s}^{2}$. The most common source of shock in portable electronics is a drop to the floor or bump against a hard surface. According to accepted theory [4], an "impact" shock pulse to a product must have a critical velocity change and a critical deceleration in order for a component inside the product to fail.

There are a few important factors that determine how this shock event is transmitted to the electronic device. The primary factors are drop height, type of impact surface, product's materials and configuration of the components in electronic device. Often a one-degree of freedom model (one DOF) of a spring-mass-damper is used to describe the effects of the shock by assuming the device-under-test (DUT) is restricted in just a vertical free drop direction. Its allow simple and straightforward solutions to be made for subsequent analysis. In summary, the common assumptions used are:

1. One DOF model of a spring-mass-damper is used.

2. Chasis of the electronic devices is infinitely rigid.

3. Shock pulse is transferred directly into component of concern inside the product.

4. DUT is in fully assembled condition.

5. The shock pulse response is a half sine wave.

\subsection{Derivation of free drop dynamic}

The primary cause of damage to any electronic devices is the high shock level experiences during the free drop. The primary factor that determines $\mathrm{G}$ level in free drop is the drop height, $H$ and rebound, $\psi$. It characterizes how the dropped object changes velocity at the moment of impact. For full rebound $\psi=2$, partial rebound $1<\psi<2$ and with no rebound $\psi=1$. Since most objects do not exhibit behaviour at the extremes, using a partial rebound value of $\psi=1.5$ is appropriate for most equipment. The derivation of the one DOF free drop from $H$ with the rebound $\psi$ is shown in the next section.

By using conservation of energy method, the kinetic (K.E) and potential energy (P.E) at the initial and final impact can be shown in Fig. 1.

The change in velocity, $\Delta V$ at the moment of impact from a drop height of $\mathrm{H}$ with a partial rebound $\psi$ yields:

$$
\begin{aligned}
{ }_{2}^{1} m \Delta V^{2} & =m g H \\
\Delta V & =\sqrt{ } 2 g H
\end{aligned}
$$

With $\psi$ gives:

$$
\Delta V=\psi \sqrt{ } 2 g H
$$

In the above equation, $g$ is the gravitational constant equal to $9.81 \mathrm{~m} / \mathrm{s}^{2}$. Using the assumption 5 , the following formula holds for acceleration:

$$
A=\frac{\Delta V}{t_{e}}=\begin{gathered}
\psi \sqrt{ } 2 g H \\
t_{e}
\end{gathered}
$$

The effective duration, $t_{e}$ of a shock pulse that is dependent upon the component shape and duration must be known before the above equation can be solved. For a half sine pulse, the effective duration is: 


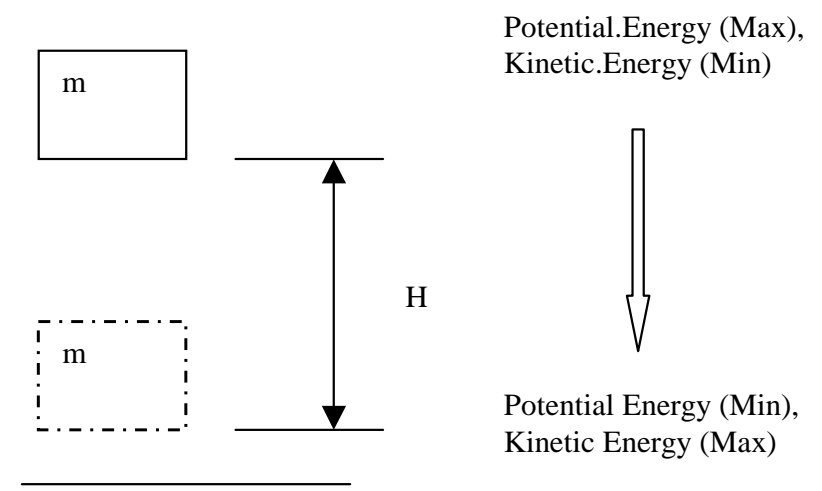

Fig. 1. Schematic diagram of free drop.

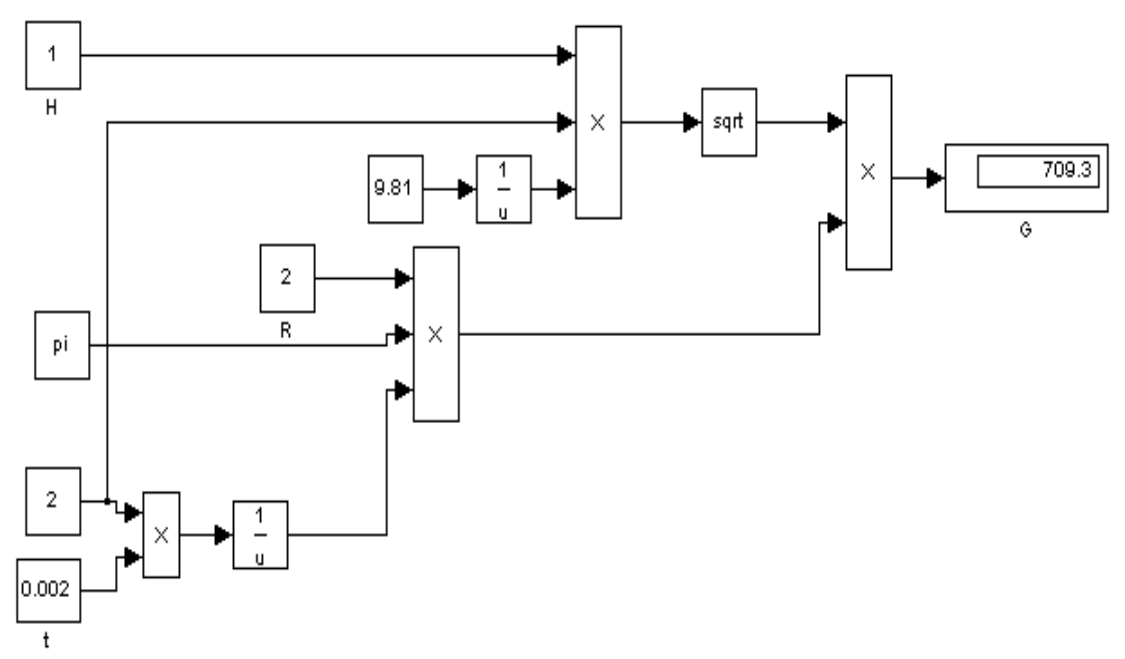

Fig. 2. Energy conservation model using MATLAB/SIMULINK.

$$
t_{e}={ }_{\pi}^{2} t
$$

Substituting Eq. (4) into Eq. (3) and convert the acceleration value to Gs, yields the shock due to free drop at height, $\mathrm{H}$, rebound, $\psi$ and pulse width, $\mathrm{t}$ :

$$
G=\begin{gathered}
\pi \psi \sqrt{ } 2 g H \\
2 t g
\end{gathered}=\pi \psi\left(\begin{array}{c}
2 H \\
g
\end{array}\right.
$$

The free drop model using MATLAB/SIMULINK [6] is shown in Fig. 2.

\subsection{Derivation of tilt tester dynamic}

The component is mounted onto the clamp as shown in Fig. 10. The derivation of one DOF tilt drop from angle, $\theta$ on plywood using Newton's second law is shown. Applying Newton second law:

$$
I \ddot{\theta}+m g R \sin \theta+b \dot{\theta}=T_{c}
$$




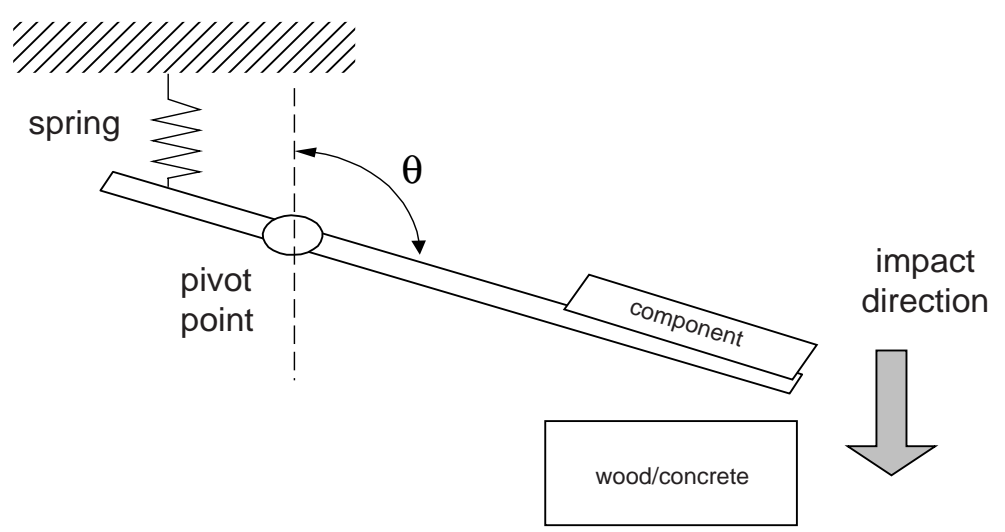

Fig. 3. Tilt tester.

where $I$ is the inertia, $m=0.018 \mathrm{~kg}$ is the mass of the component, $R=0.067 \mathrm{~m}$ is the length of the arm, $T_{c}$ is the torque, and $b=10 \mathrm{Ns} / \mathrm{m}$ is the damping constant. Substituting $I=m R^{2}$ and $T_{c}=G g R$ gives:

$$
m R^{2} \ddot{\theta}+m g R \sin \theta+b \dot{\theta}=G_{i} g R
$$

where $\mathrm{G}_{i}$ is the desired shock level in $g$ exerted on the component. Rearranging Eq. (7):

$$
\ddot{\theta}+{ }_{R}^{g} \sin \theta+{ }_{m R^{2}}^{b} \dot{\theta}=\begin{gathered}
G_{i} g \\
m R
\end{gathered}
$$

In state-space form, for $x_{1}=\theta, x_{2}=\dot{\theta}$ :

$$
\dot{x}_{1}=\dot{\theta}, \dot{x}_{1}=x_{2}, \dot{x}_{2}=\ddot{\theta}, \dot{x}_{2}=\begin{gathered}
G_{i} g \\
m R
\end{gathered}-{ }_{m R^{2}}^{b} x_{2}-{ }_{R}^{g} \sin x_{1}
$$

In matrix form, Eq. (9) becomes:

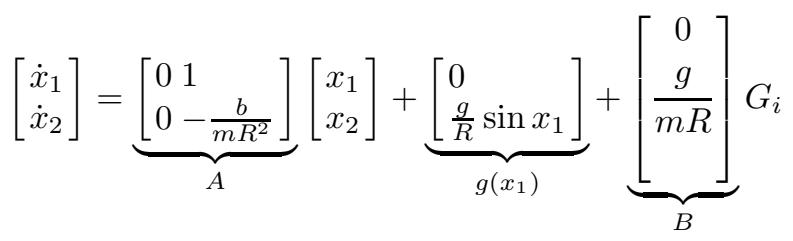

By selecting the desired input, $G_{i}$ in Eq. (10), results in the tilt angle $\theta$. The tilt tester model using MATLAB/SIMULINK [6] is shown in Fig. 4.

\subsection{Validate free drop and tilt test dynamic model}

Using drop orientation in $\mathrm{x}, \mathrm{y}$ and $\mathrm{z}$ direction, a MP3 player was dropped at various height $(5-30 \mathrm{~cm})$ to determine the shock levels on the product. Applying the energy conservation principle in Eq. (5) on the MP3 player (using H $=5 \mathrm{~cm}$ and assuming $\psi=1.5, t=1 \mathrm{~ms}$ ) gives $237 \mathrm{G}$.

As observed in Fig. 5 , at $5 \mathrm{~cm}$ free drop on plywood, "best" approximate the shock level of $237 \mathrm{G}$ (as calculated above) on the component. Note that variability in the shock level is smaller (as shown in Fig. 7) at lower drop height. This can be explained since orientation at point of impact can be easily aligned at lower height.

Besides the free drop test, tilt test on the component was conducted. Applying Eq. (7) on the component, the shock level at various tilt angles can be shown in Fig. 7. As observed, the tilt angle increases as shock level (in G) increases. Since it is difficult to solve Eq. (7) analytically, the numerical model in Fig. 4 is used to calculate the angle for $G_{i}=237 \mathrm{G}$. Comparing the experimental results in Fig. 7 with the numerical simulation in Fig. 6, it shows good approximation of the tilt tester model (at tilt angle of $60^{\circ}$ ). 

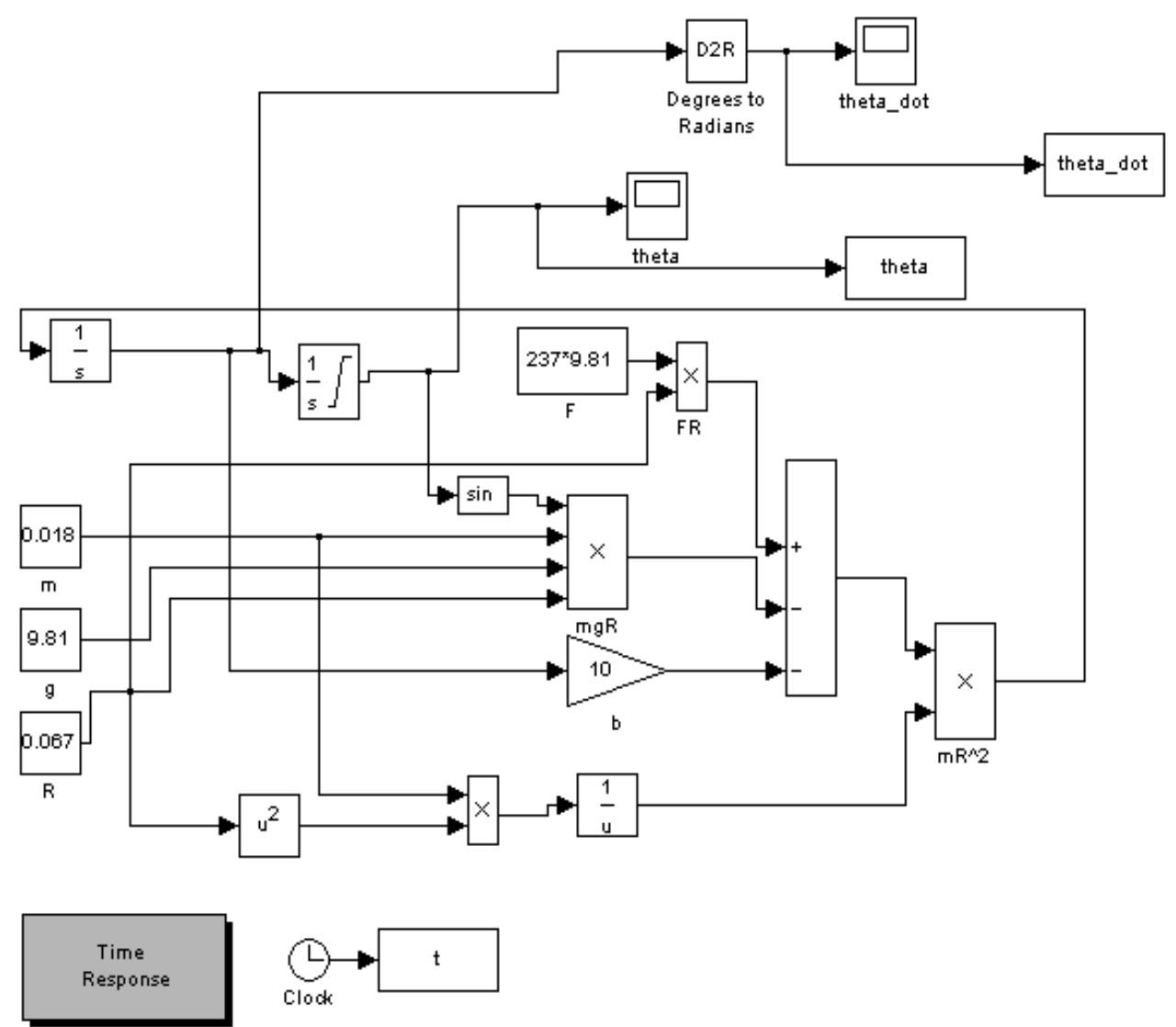

Fig. 4. Tilt tester model using SIMULINK.

\subsection{Correlate tilt tester with free drop test}

As shown above, to achieve about $237 \mathrm{G}$ (for $5 \mathrm{~cm}$ drop height), the equivalent tilt angle, $\theta=60^{\circ}$. As computed through a statistical software MINITAB, shock level variation (in percentage) in the tilt tester is approximately $5.8 \%$ as compared to the free drop, which can be greater than $30 \%$. Thus, it shows that the tilt tester has better repeatability than the free drop. Therefore, instead of dropping the MP3 player, a corresponding test using the tilt tester can be used. In subsequent section, it can be seen that the improvement in shock level as seen in tilt tester can be observed from the free drop test as well. The next steps are to use the tilt tester to design a shock absorber for reducing the shock exposure of the component embedded in MP3 player.

\section{Shock absorber design}

After correlating the tilt test with the free drop, a systematic design of a shock absorber to reduce the shock level of the component embedded in the product is shown. In Fig. 8, after the free drop test on the MP3 player, improvement on the component embedded in the MP3 player is required if the shock level exceed a specified value. Instead of performing multiple tests on a fresh MP3 player, the component and the original shock absorber was took out and placed inside the clamp of the tilt tester (made of Thermoplastic material that simulate the actual enclosure that the product experience under embedded condition) as shown in Fig. 9. Instead of trial and error means of selecting the shock absorber, a systematic step to select a suitable shock absorber is performed. Once the desired shock level 
Multi-Vari Chart for Shock Level by Height

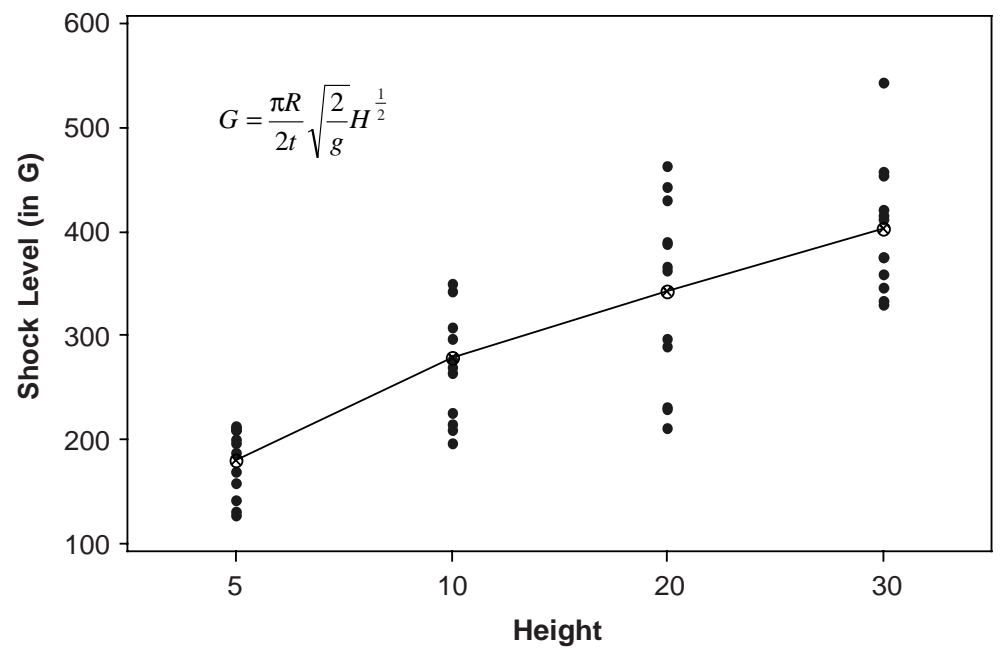

Fig. 5. Component's shock response in MP3 player due to free drop.

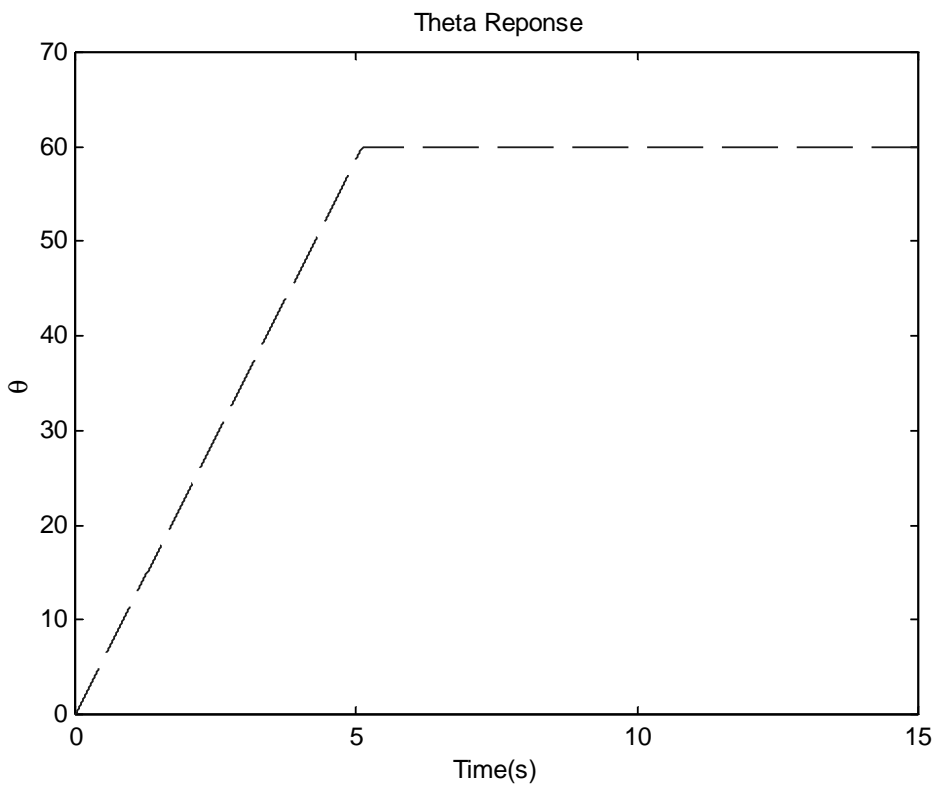

Fig. 6. Angle for $\mathrm{G}=200 \mathrm{~g}$.

(within the customer's specification) is achieved, the component with the new shock absorber has to go through a validating test using the free drop.

There are plenty of shock absorbers such as Vinyl Thermoplastic, Thermoplastic Ethane, Low Modulus Urethane, High Density Urethane Elastomer and Urethane Foams available in the markets. It is vital to select shock absorber that can meet the requirement of the shock level specified by customer. The steps in the design and placement of shock absorber are shown. 
Individual Value Plot of Shock Value at 30, 45, 60, 75, 90 Degree

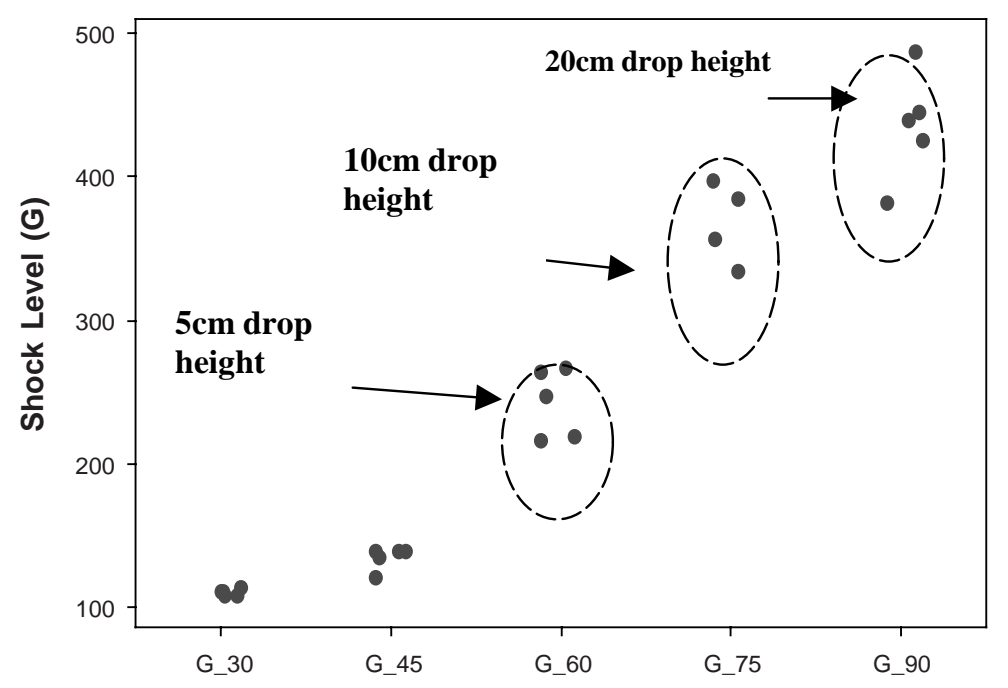

Fig. 7. Shock level due to free drop.

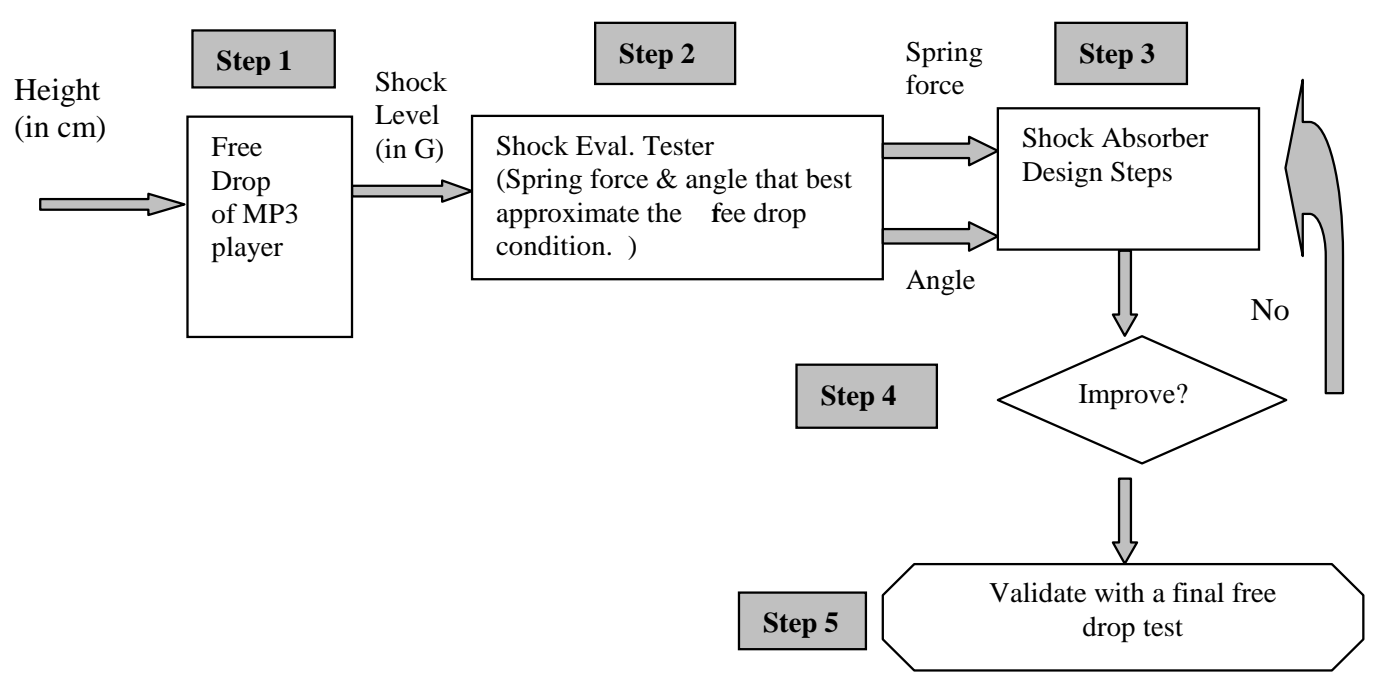

Fig. 8. Shock testing flow chart using tilt tester.

\subsection{Optimize pressure load}

Overloading an elastometric mount can reduce its useful life. Generally, a 5\% static compression of the mount is appropriate for most materials. For homogeneous elastomers [3] with a durometer (hardness) of around 50-60 shore A, ideal loading is about $30 \mathrm{MN} / \mathrm{m}^{2}$ [1,2]. Softer elastomers should be loaded less than stiffer elastomers.

By assuming no other external force exerted on the component (with a surface area $=36.4 \mathrm{~mm} \times 42.8 \mathrm{~mm}$ $\left.=1.557 \times 10^{-3} \mathrm{~m}^{2}\right)$ except a specified force requirement of $2000 \mathrm{G}(=20 \mathrm{KN})$ that the shock absorber has to withstand during the drop test. This gives a maximum pressure on the component (in $\mathrm{MN} / \mathrm{m}^{2}$ ) to be $20 \times$ $10^{3} / 1.557 \times 10^{-3}=13 M N / m^{2}$. 


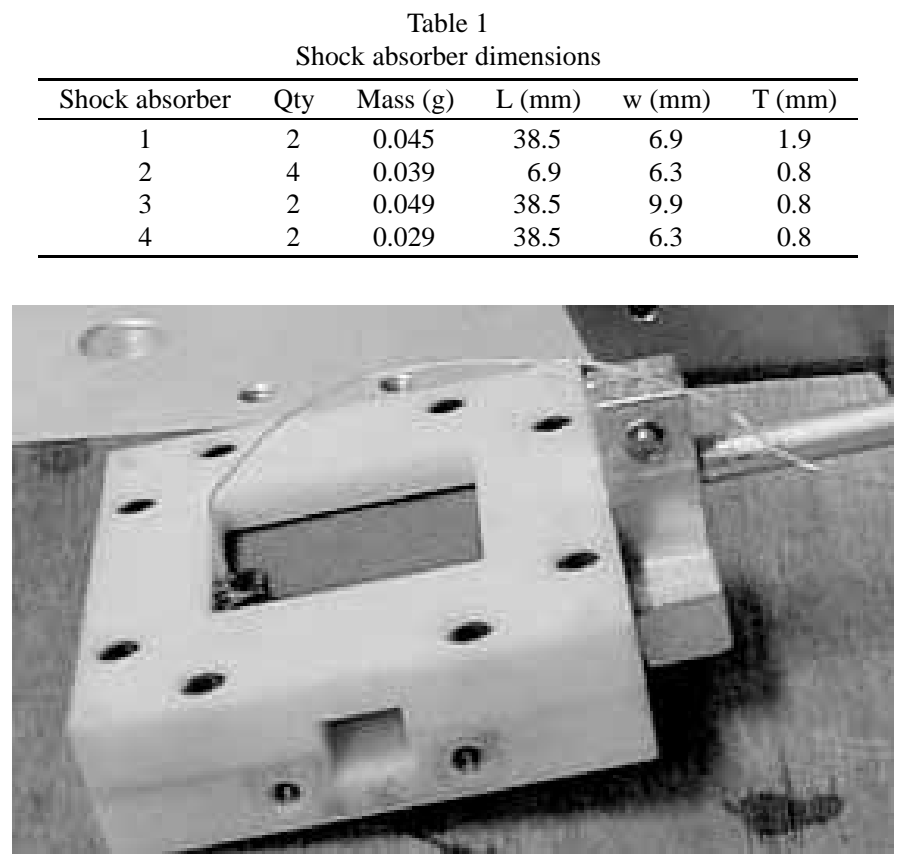

Fig. 9. Clamp of the tilt tester for shock absorber testing.

\subsection{Dimensions of the shock absorber}

By separating the shock absorber into four parts as shown in Fig. 10, the dimensions for each part are tabulated in Table 1.

\subsection{Shape factor $(S)$}

Solid elastomers act as incompressible solids, and therefore must have room to bulge in order to deflect. The shape or bulge factor should be optimized to achieve the expected stiffness. A high S produces a stiff mount and low $\mathrm{S}$ may buckle and be unstable. Shape factor $(\mathrm{S})$ is defined as:

$$
S=\text { Area under load }
$$

By applying the Eq. (11) in $x, y$ and $z$ direction as shown in Fig. 10, a rectangular shaped shock absorber with a maximum size of $38.5 \mathrm{~mm} \times 6.3 \mathrm{~mm} \times 0.8 \mathrm{~mm}$, the shape factor:

$$
S_{x}=\begin{gathered}
38.5 \times 6.3 \\
2(6.3 \times 0.8+38.5 \times 0.8)
\end{gathered}=3.38
$$

A rectangular shaped shock absorber with a maximum size of $6.9 \mathrm{~mm} \times 6.3 \mathrm{~mm} \times 0.8 \mathrm{~mm}$, the shape factor:

$$
S_{y}=\begin{gathered}
6.9 \times 6.3 \\
2(6.3 \times 0.8+6.9 \times 0.8)
\end{gathered}=2.06
$$

A rectangular shaped shock absorber with a maximum size of $38.5 \mathrm{~mm} \times 9.9 \mathrm{~mm} \times 1.9 \mathrm{~mm}$, the shape factor:

$$
S_{z}=\begin{gathered}
38.5 \times 9.9 \\
2(9.9 \times 0.8+38.5 \times 0.8)
\end{gathered}=4.92
$$

As shown in Fig. 10, there are two dampers in $\mathrm{Z}$ direction, two dampers in $\mathrm{X}$ direction and one in $\mathrm{Y}$ direction, the average shape factor:

$$
\begin{gathered}
3.38+2.06+4.92 \\
2+2+1
\end{gathered}=2.072
$$




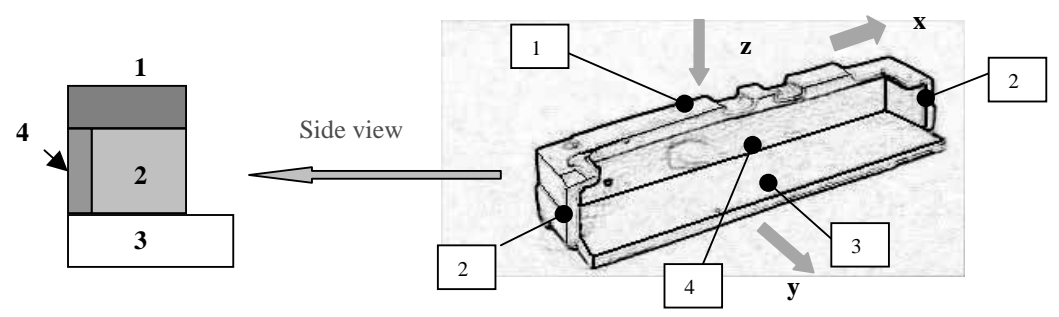

Fig. 10. Block 1, 2 and 3 in series and parallel with 4.

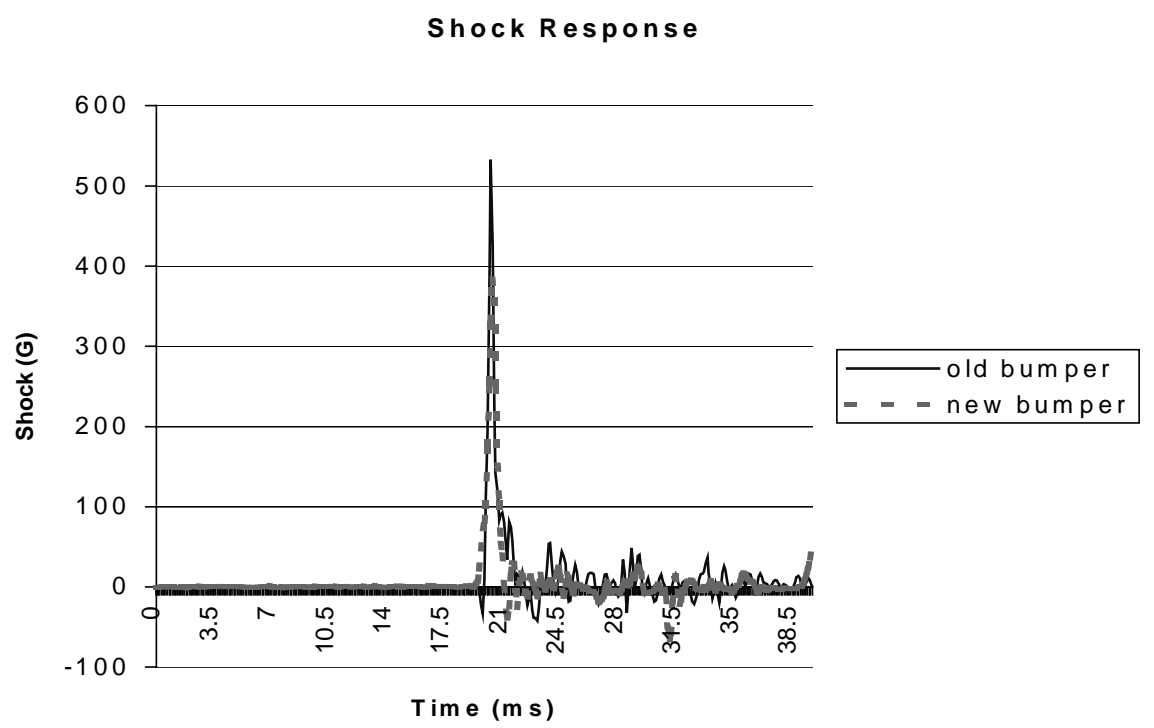

Fig. 11. Shock level due to tilt test using old and new shock absorber.

\subsection{Dynamic modulus of elastomeric $\left(E_{\text {corrected }}\right)$}

The dynamic modulus of the material can be determined using the formulae below. For a block shaped shock absorber, the corrected dynamic modulus of Elastomeric:

$$
E_{\text {corrected }}=4 / 3 E\left(1+2 S^{2}\right)
$$

By assuming the shock absorber made of general Silicone rubber compounds, the corrected dynamic modulus of Elastomeric:

$$
\begin{aligned}
E_{\text {corrected }} & =4 / 3 E\left(1+2 S^{2}\right) \\
& =4 / 3 \times 896 \times 10^{3}\left(1+2 \times 2.072^{2}\right)=11.45 \mathrm{MN} / \mathrm{m}^{2}
\end{aligned}
$$

\section{5. $\operatorname{Stiffness}(K)$}

Calculate stiffness of the shock absorber using the following formulas. For a disk shaped shock absorber, the stiffness:

$$
K_{s}=\frac{E_{\text {corrected }} \pi r^{2}}{t}
$$

For a block shaped shock absorber, the stiffness: 


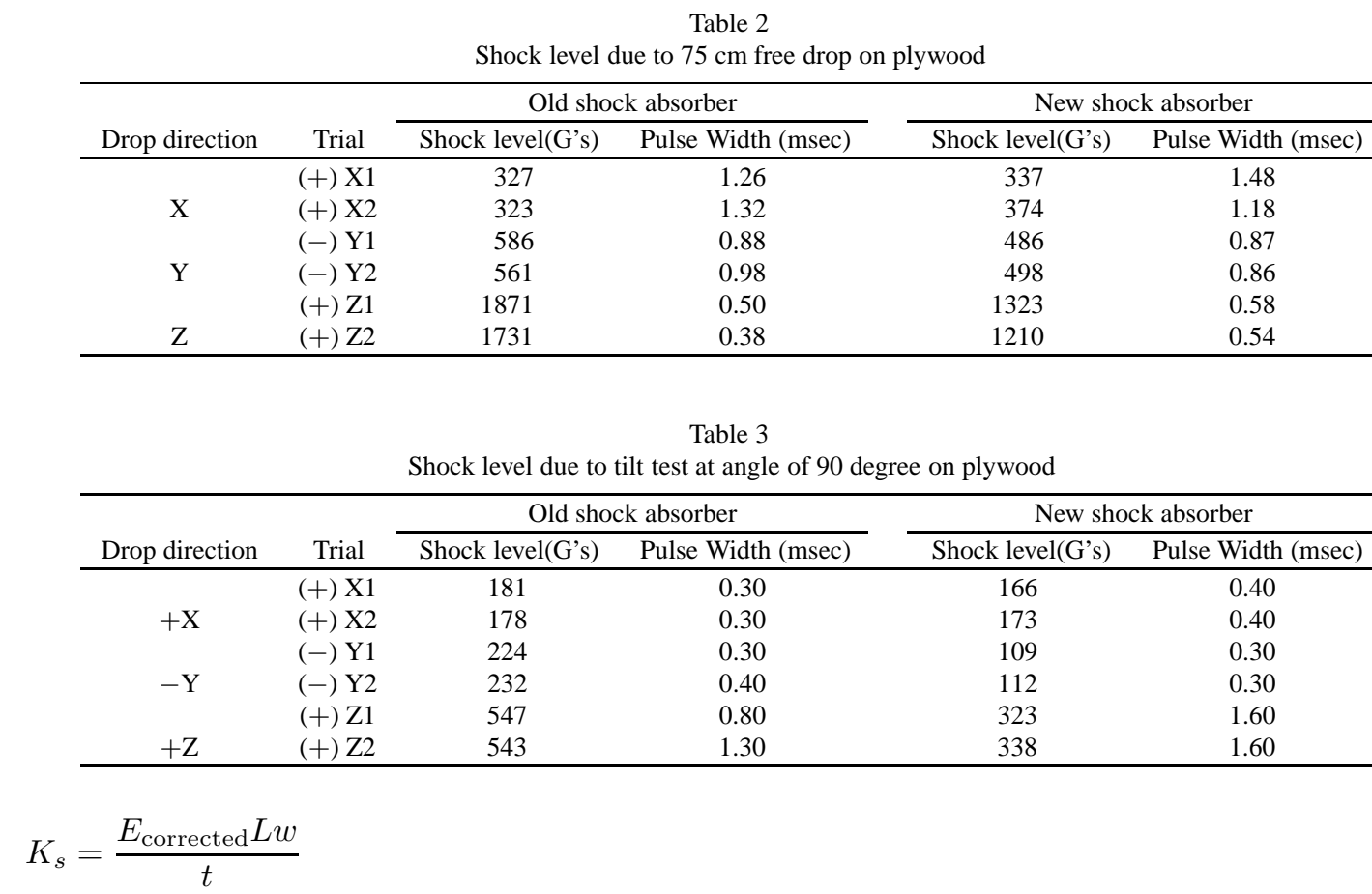

where $r$ is the disk radius, $L$ is the block width, $t$ is the thickness and $w$ is the block width. The shock absorber of different shape and locations are combined to achieve certain shock absorber capability, hence the rule of electrical resistance in series and parallel can be applied. The total stiffness of the shock absorber:

$$
\begin{aligned}
K_{\text {total }}= & \underset{{ }_{K_{4}}}{1}+2 \underset{K_{2}}{1}+K_{1}+K_{3} \\
= & 11 \underset{3.5}{1}+\underset{0.62}{2}+1.05+1.6=2.93 M N / m
\end{aligned}
$$

After the shock absorber is selected, evaluation of the shock level on the component using the tilt tester is performed. The shock response of the component with the shock absorber is shown in the following section.

\section{Results of shock response}

With the use of the original shock absorber, the shock level (obtained using an Endevco [8,9] tri-axial accelerometer) due to free drop (from $75 \mathrm{~cm}$ on plywood) at $x, y$ and $z$ drop direction is shown in Table 2.

By using data acquisition software, Test Partner 3 [7] (TP3) through a signal conditioner, shock responses of the component using old and new absorber are shown in Fig. 11. Besides, the shock response spectrum (SRS) [1] method that is an excellent method for characterizing the acceleration response of a system is used. As shown in Fig. 12, peak acceleration occurs at $1050 \mathrm{~Hz}$ for component using both old and new shock absorber.

The shock level is high prior to use of new shock absorber. Using the tilt tester (at 90 degree tilt angle drop on plywood) to evaluate the performance of the new shock absorber, improvement in shock level was observed at all axes (see Table 3). This was verified with the free drop test and shown to have improvement in y's and z's drop directions. This facilitates the evaluation of the shock absorber without having to perform multiple drops using the MP3 player. The cost and test time are reduced.

By correlating the improvement using the tilt tester to the free drop, both show reduction in shock level in $\mathrm{Y}$ and $\mathrm{Z}$ drop direction but no improvement in $\mathrm{X}$. 


\section{Shock Response Spectrum}

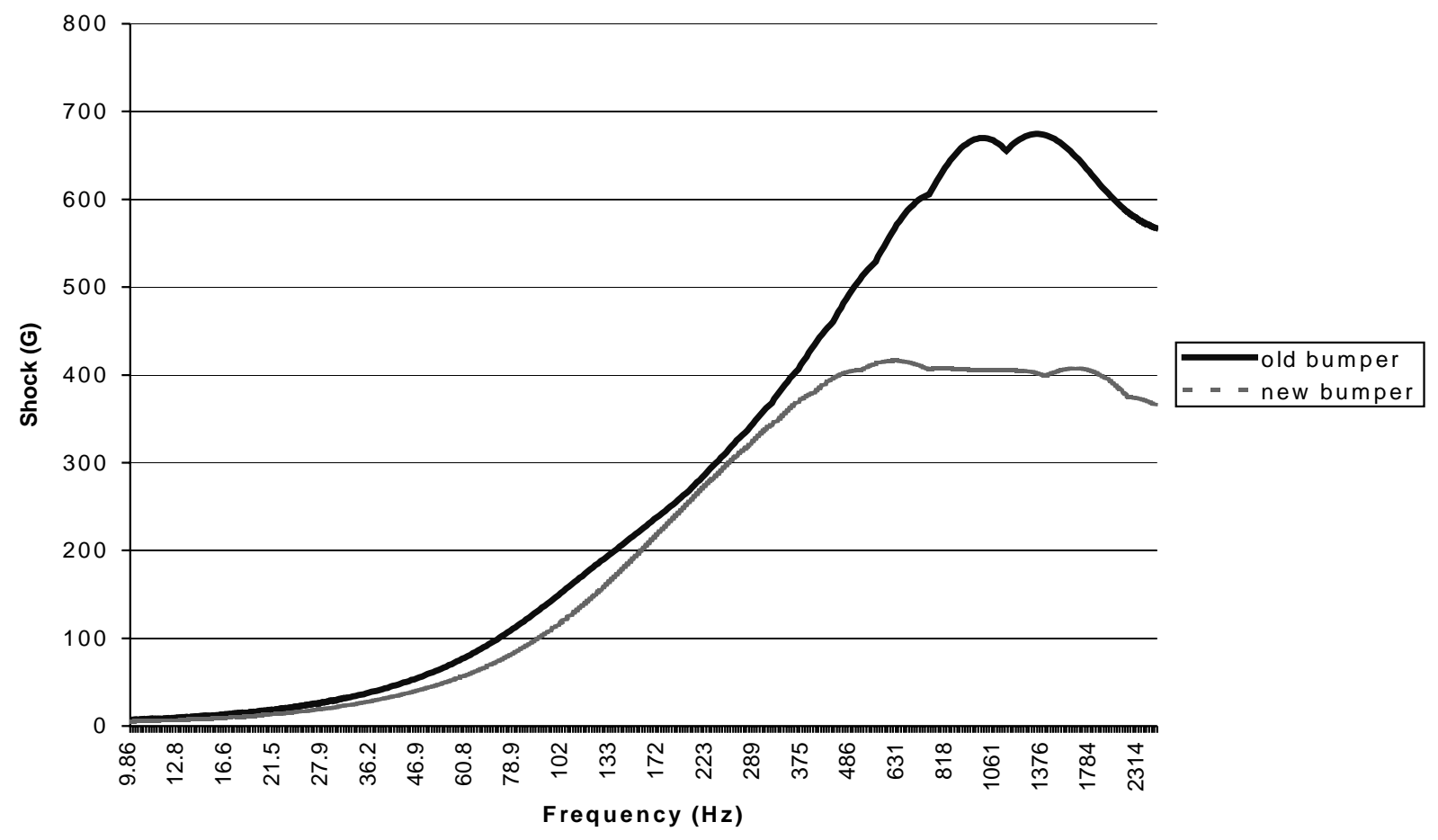

Fig. 12. Shock response spectrum (SRS) for old and new shock absorber.

\section{Conclusions}

Dynamic model due to free drop and tilt tester using energy conservation and Newton's second law were derived. Comparison with the shock table test was made. Since the intent of lab testing is try to re-create the real world events, a unique ways to establish a test that encompasses the features the above free drop and shock table test is needed. A high repeatable and realistic shock testing using tilt tester was designed for component embedded in MP3 player.

The validity of this tilt tester was made using experimental results of the component (free drop on a plywood platform). Results shown to have a good approximation to the actual free drop testing. The systematic design steps to facilitate shock absorber design were performed. This ensures that improvement in shock level is found before conducting a final free drop test with component embedded in MP3 player. This reduces the number of trials and products needed for testing. Demonstrating this on the component, the shock reduces from $2000 \mathrm{G}$ to $1100 \mathrm{G}$ and the cycle time in testing reduces.

\section{Acknowledgements}

This work is also assisted by Mr Steven Goh and grateful for his guidance and support. And thank the anonymous reviewers, whose suggestions were instrumental in improving the final version of the paper.

\section{References}

[1] I. Daniel, Engineering Vibration, Englewood Cliffs, N.J., Prentice Hall Inc, 1994. 
[2] L. Harold, W. Gatley and H. Evensen, Noise Control for Engineers, New York, McGraw Hill Book Company, 1980.

[3] G. Ross, The Reduction of High Frequency Vibration \& Noise by using E-A-R ISODAMP Isolation Materials, Indianapolis: E-A-R Division, Cabot Coporation, 1976.

[4] Newton, E. Robert, Fragility Assessment of Theory \& Test Procedure, US Naval Postgraduate School.

[5] Selected ASTM Standards on Packaging, Fourth Edition, Philadelphia, P. A, ASTM, 1994.

[6] E.P. Enander and A. Sjoberg, The Matlab 5 Hand Book, Addison Wesley, 1999.

[7] Test Partner 3, Lansmont Corporation, 2003.

[8] K.S. Hansen, A New Design Principle for Tri-Axial Piezoelectric Accelerometer, SENSOR 1997.

[9] K.S. Hansen, A New Design Principle for Tri-Axial Piezoelectric Accelerometer, $8^{\text {th }}$ International Congress on Sound \& Vibration, Hong Kong, 2001. 

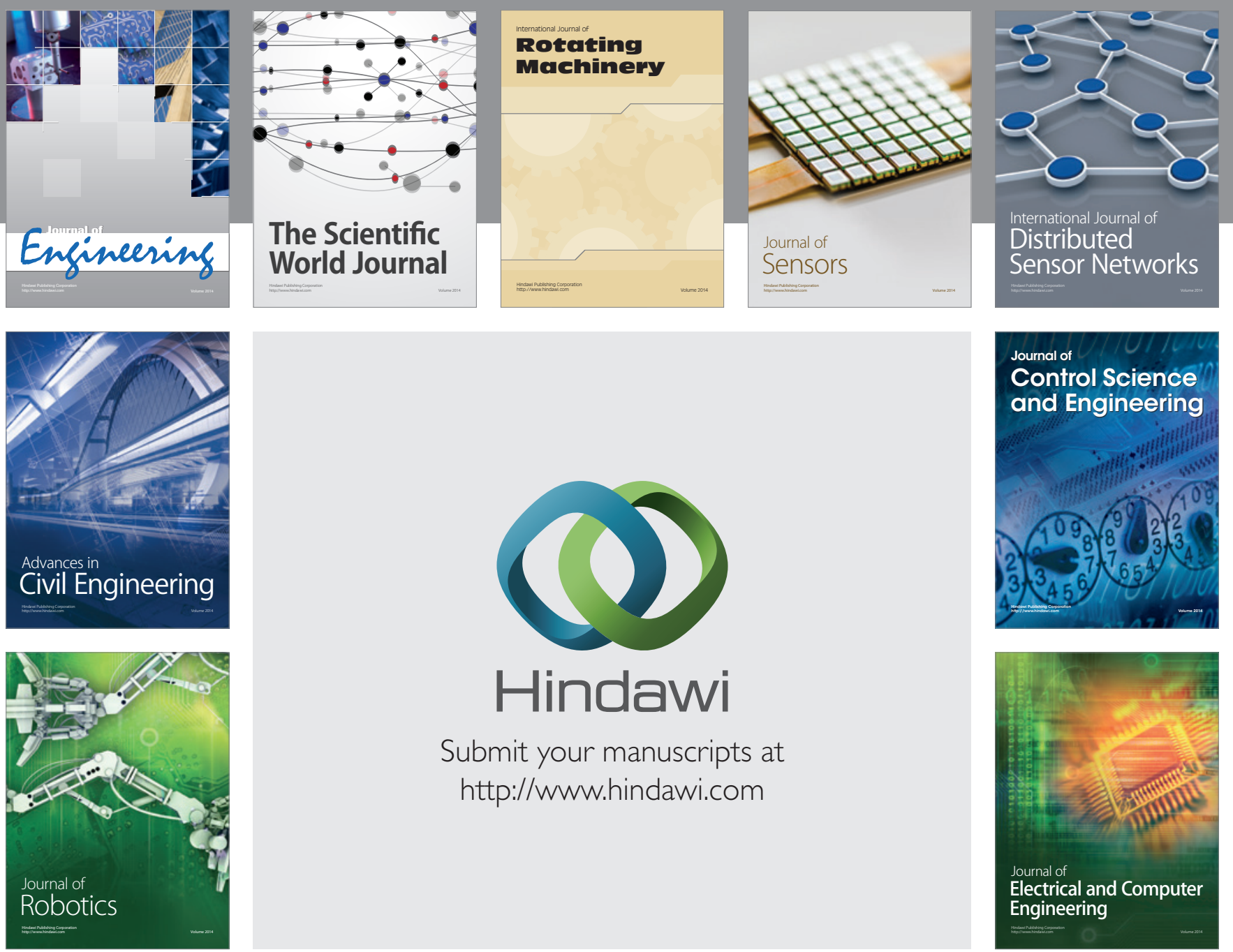

Submit your manuscripts at

http://www.hindawi.com
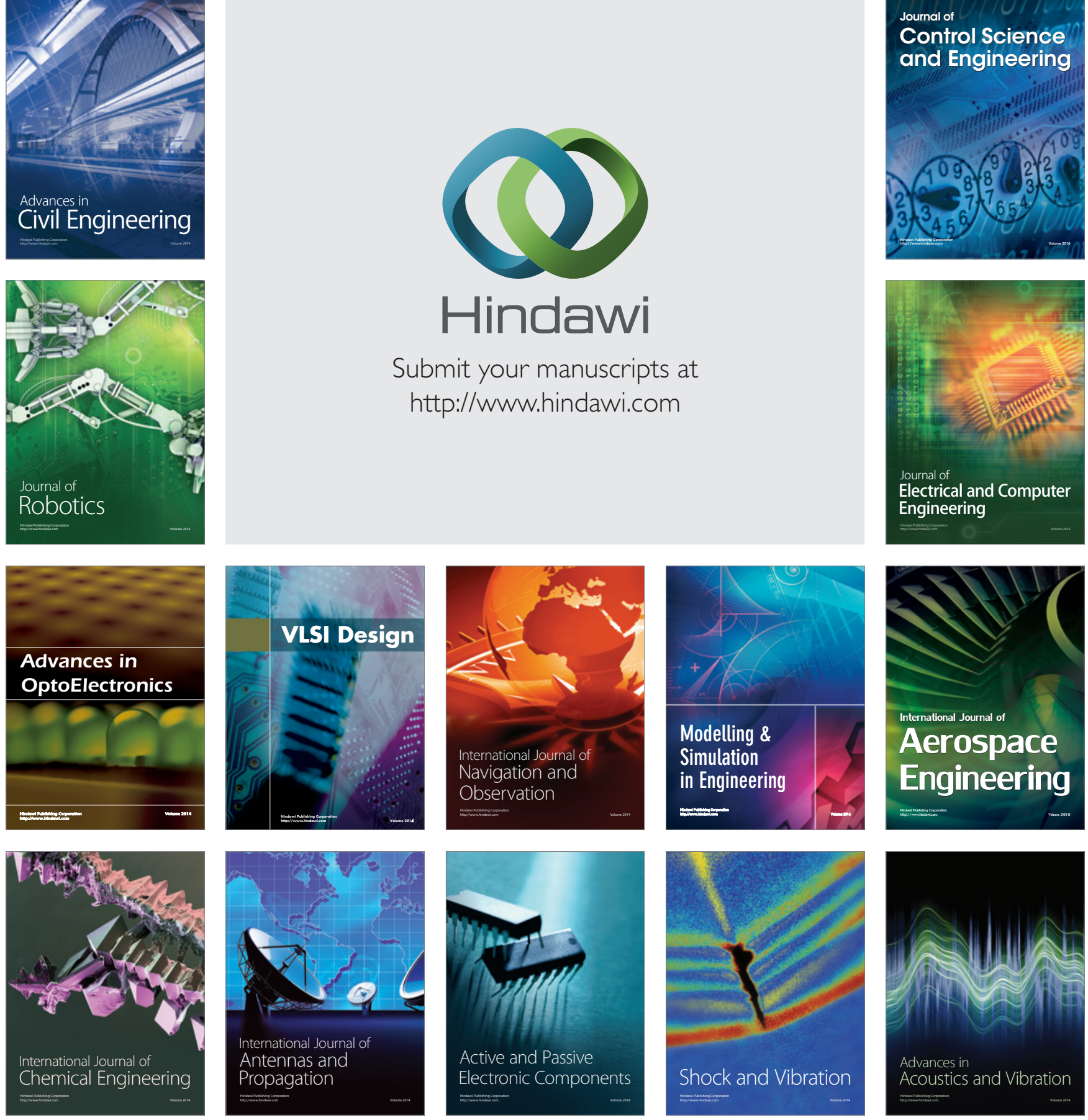